INNATE IMMUNITY

\section{Neutrophil recruitment on the big screen}

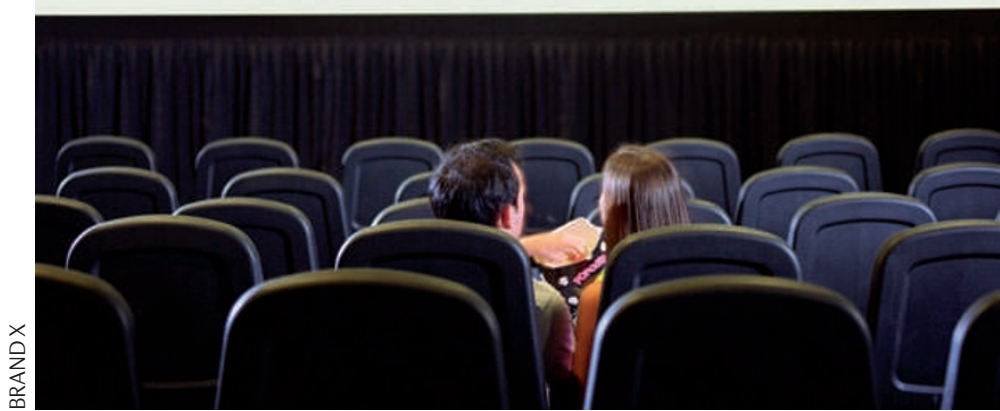

In a recent study published in Science, Paul Kubes and colleagues describe a multistep cascade that guides neutrophils through the circulation to sites of sterile inflammation in vivo.

Tissue injury in the absence of infection (sterile inflammation) results in the recruitment of neutrophils from the blood to the tissue site, where they contribute to wound healing but may also cause tissue damage if not controlled. To understand the mechanisms involved in neutrophil recruitment in vivo, the authors generated a mouse model of focal hepatic necrosis using localized thermal injury on the surface of the liver. They then imaged the recruitment of endogenous neutrophils (expressing green fluorescent protein) to the necrotic lesion (visualized by superfusion of the injured site with propidium iodide) using spinning disk confocal intravital microscopy.

The authors found that within 30-60 minutes of injury, neutrophils started adhering to the microvascular endothelium around the site of injury. This adhesion was mediated by $\alpha \mathrm{M} \beta 2$ integrin and intercellular adhesion molecule 1 (ICAM1).
By contrast, when Escherichia coli were applied to the liver surface in the absence of thermal injury, neutrophil adhesion in the sinusoids depended on the adhesion molecule $\mathrm{CD} 44$ and not $\alpha \mathrm{M} \beta 2$ integrin, suggesting distinct mechanisms of neutrophil recruitment in response to sterile inflammation and infection. At 2-3 hours after injury, neutrophils accumulated within

What is the signal from the necrotic lesion that initiates this recruitment? ATP released from the necrotic cells was shown to activate the NLRP3 (NOD-, LRR- and pyrin domain-containing 3) inflammasome through P2X7 receptor signalling in non-neutrophil haematopoietic cells (probably Kupffer cells). This resulted in interleukin-1 $\beta$ (IL-1 $\beta$ ) production and upregulation of ICAM1 expression on the surface of the sinusoidal endothelium, and subsequent $\mathrm{\alpha M} \beta 2$ integrinICAM1-mediated neutrophil adhesion in liver sinusoids around the site of injury. However, this pathway did not guide neutrophils into the sterile necrotic lesion. the necrotic lesions.
Further analysis showed that neutrophil chemotaxis into the sterile necrotic lesion required myeloid differentiation primary response protein 88 (MYD88)-dependent signalling in non-haematopoietic cells (but was independent of IL-1 $\beta$ ). These signals resulted in the production of an intravascular gradient of CXC-chemokine ligand 2 (CXCL2) that was maximal at $\sim 100-150 \mu \mathrm{m}$ from the injury border and decreased outwards. However, within the area immediately surrounding the injury site (up to a distance of $150 \mu \mathrm{m}$ ), neutrophil migration was CXCL2-independent. So what mediates migration through non-perfused sinusoids into the necrotic lesion? The authors showed that necrotic cells released a 'necrotaxis' signal in the form of mitochondria-derived formylated peptides. This signal specifically directed neutrophil chemotaxis into the injury site, mediated by formyl peptide receptor 1 signalling.

So, ATP released following sterile injury activates the NLRP3 inflammasome in tissue-resident cells to generate the inflammatory environment that mediates neutrophil adhesion in the local vasculature. A CXCL2 gradient then guides neutrophils through the sinusoids to the necrotic lesion; this intravascular recruitment could help to protect against collateral damage of the local healthy tissue. Finally, necrotaxis signals direct neutrophils from the non-perfused sinusoids into the sterile injury site. Interestingly, a similar set of cues guides neutrophils to sites of sterile inflammation in the skin, highlighting the general importance of this multistep pathway.

Olive Leavy

ORIGINAL RESEARCH PAPER McDonald, B. et al. Intravascular danger signals guide neutrophils to sites of sterile inflammation. Science 330, 362-366 (2010)

FURTHER READING Chen, G. Y. \& Nuñez, G. Sterile inflammation: sensing and reacting to damage. Nature Rev. Immunol. 10, 826-837 (2010) 